\title{
Magnetomotive laser speckle imaging
}

\author{
Jeehyun Kim \\ Kyungpook National University \\ Electrical and Computer Engineering \\ 1370 Bukgu San-geok dong \\ Daegu \\ South Korea
}

\author{
Junghwan Oh \\ Pukyong National University \\ Mechanical Engineering Department \\ San 100 Yongdang dong, Nam Gu \\ Busan, 608-739 \\ South Korea
}

\author{
Bernard Choi \\ University of California, Irvine \\ Beckman Laser Institute and Medical Clinic \\ 1002 Health Sciences Road East \\ Irvine, California 92612 \\ and \\ University of California, Irvine \\ Department of Biomedical Engineering \\ 3120 Natural Sciences I \\ Irvine, California 92697
}

\begin{abstract}
Laser speckle imaging (LSI) involves analysis of reflectance images collected during coherent optical excitation of an object to compute wide-field maps of tissue blood flow. An intrinsic limitation of LSI for resolving microvascular architecture is that its signal depends on relative motion of interrogated red blood cells. Hence, with LSI, small-diameter arterioles, venules, and capillaries are difficult to resolve due to the slow flow speeds associated with such vasculature. Furthermore, LSI characterization of subsurface blood flow is subject to blurring due to scattering, further limiting the ability of LSI to resolve or quantify blood flow in small vessels. Here, we show that magnetic activation of superparamagnetic iron oxide (SPIO) nanoparticles modulate the speckle flow index (SFI) values estimated from speckle contrast analysis of collected images. With application of an ac magnetic field to a solution of stagnant SPIO particles, an apparent increase in SFI is induced. Furthermore, with application of a focused dc magnetic field, a focal decrease in SFI values is induced. Magnetomotive LSI may enable wide-field mapping of suspicious tissue regions, enabling subsequent high-resolution optical interrogation of these regions. Similarly, subsequent photoactivation of intravascular SPIO nanoparticles could then be performed to induce selective photothermal destruction of unwanted vasculature. (0) 2010 Society of PhotoOptical Instrumentation Engineers. [DOI: 10.1117/1.3285612]
\end{abstract}

Keywords: contrast agents; laser Doppler flowmetry; perfusion; speckle contrast.

Paper 09336SSR received Aug. 5, 2009; revised manuscript received Oct. 29, 2009; accepted for publication Nov. 2, 2009; published online Jan. 13, 2010.

\section{Introduction}

The method of laser speckle imaging (LSI) involves analysis of raw speckle reflectance images collected during coherent optical excitation of an object. Spatial, ${ }^{1}$ temporal, ${ }^{2}$ and spatiotemporal $^{3}$ algorithms are used to convert these images into maps of speckle contrast. Based on an assumption of the line shape of the correlation function, ${ }^{4}$ the speckle contrast maps are then converted to maps of relative blood flow. Camera exposure time is used to select the flow rate sensitivity and linear response range of LSI, ${ }^{5}$ and use of multiple exposure times during data collection can extend this range. ${ }^{6}$

Previous studies have demonstrated the practical equivalence of LSI and laser Doppler flowmetry (LDF). ${ }^{1-7}$ Advantages of LSI instruments over commercial LDF systems include simultaneous high spatial and temporal resolution, ease of implementation, and relatively low cost. Due in large part to these advantages, LSI has been used in a myriad of applications for blood flow characterization, including: 1. study of blood flow dynamics associated with laser therapy of the microvasculature, ${ }^{8-10} 2$. study of blood flow dynamics associated with focal cerebral ischemia, ${ }^{1,11,12} 3$. study of arteriolar and venular flow dynamics in response to optical clearing agent application, ${ }^{5,13}$ and 4 . study of tissue biomechanics with speckle motion tracking. ${ }^{14}$

Address all correspondence to Bernard Choi, Univ. of California-Irvine, Beckman Laser Institute, 1002 Health Science Rd. E, Irvine, CA 92612; Tel: 949 824-9491; Fax: 949 824-6969; E-mail: choib@uci.edu
With appreciable blood flow in superficial vasculature, it is possible to use LSI images to characterize microvasculature parameters such as vessel diameter. However, similar to Doppler optical coherence tomography (OCT), ${ }^{15,16}$ an intrinsic limitation of LSI for resolving microvascular architecture is that its signal depends on the relative motion of the interrogated scatterers (i.e., red blood cells). Hence, with LSI, smalldiameter arterioles, venules, and capillaries are difficult to resolve due to the slow flow speeds associated with such vasculature. Furthermore, LSI characterization of subsurface blood flow is subject to blurring due to optical scattering, further limiting the ability of LSI to resolve or quantify blood flow in small vessels. The presence of static optical scatterers reduces the accuracy of estimates of the speckle correlation time associated with the true motion of red blood cells. ${ }^{6,17}$ Collectively, these limitations affect our ability to image the microvasculature using LSI methods.

Several recent publications describe the use of magnetomotive methods to increase optical contrast. Anker and Kopelman ${ }^{18}$ used a rotating magnetic field to modulate the emission of exogenous fluorescent nanoprobes. Oldenburg, Gunther, and Boppart ${ }^{19}$ published the first demonstration of magnetomotive OCT. Their data suggested a 30-dB improvement in OCT contrast with magnetic actuation of magnetite microparticles in an engineered tissue construct. In a follow-up publication, Oldenburg et al. ${ }^{20}$ employed a Xenopus

1083-3668/2010/15(1)/011110/5/\$25.00 @ 2010 SPIE 

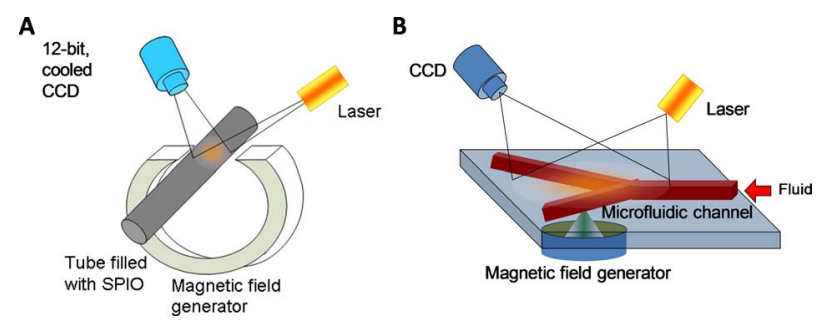

Fig. 1 Experimental setups used to (a) increase and (b) decrease motion of SPIO particles. For all experiments, we used a 633-nm HeNe laser as the coherent source and a 12-bit cooled CCD camera to image the remitted speckle pattern. (a) To increase SPIO particle motion, we used a horseshoe-shaped magnet to excite a capillary tube filled with SPIO particles, with an ac magnetic field. (b) To decrease SPIO particle motion, we used a solenoid-based magnetic field generator to induce a focused dc magnetic field within one branch of a Y-shaped channel. We used an infusion pump to induce ordered SPIO particle motion through the channel.

laevis model to demonstrate the potential in vivo application of magnetomotive OCT. Oh et al. ${ }^{21,22}$ employed magnetomotive ultrasound and OCT to study macrophage recruitment in tissue. Kim et al. ${ }^{23,24}$ employed magnetomotive principles to modulate the signal associated with Doppler OCT and hence the detectability of subsurface scatterer flow. Aaron et al. ${ }^{25}$ employed targeted nanoparticles to improve optical contrast of cells expressing epidermal growth factor receptor. Jing et $\mathrm{al}^{26}$ used cell magnetophoresis to quantify the uptake of superparamagnetic iron oxide (SPIO) particles tagged with transfection agents. Such agents are used to improve the specificity of SPIO integration into targeted cells.

We present a novel extension of the magnetomotive approach by modulating the Doppler shift to improve magnetic nanoparticle contrast in LSI. Contrast in LSI images is enhanced by activating mechanical motion of the nanoparticles with an externally applied, high-strength magnetic field gradient. We describe the magnetomotive laser speckle imaging (MM-LSI) experimental setup and present LSI images of flowing superparamagnetic iron oxide (SPIO) nanoparticles under the influence of an externally applied magnetic field gradient.

\section{Materials and Methods}

\subsection{Laser Speckle Imaging Instrument}

The LSI instrument consists of a 633-nm HeNe laser and charge-coupled device (CCD) camera (Fig. 1). The laser was transmitted through a fiber optic cable coupled to a computercontrolled attenuator and finally focused to a spot size of $4 \mathrm{~mm}$ diameter. The resultant speckle pattern was imaged with a 12-bit monochrome CCD camera (Retiga EXi, QImaging, Surrey, British Columbia, Canada) equipped with a 90-mm Elicar macrolens. To maximize speckle contrast, the $\mathrm{f} /$ number of the lens was set to achieve a minimum speckle size equal to the width of approximately two camera pixels (individual pixel pitch $=6.5 \mu \mathrm{m}) .{ }^{27}$ All image acquisition and data processing were done using custom LabVIEW software (Version 7.1, National Instruments, Austin, Texas).

\subsection{Flow Phantoms}

Capillary tube. For magnetomotive LSI experiments with an ac magnetic field, a capillary tube (inner diameter of $500 \mu \mathrm{m}$ ) filled with SPIO nanoparticles (see next section) was used as the test substrate.

Microfluidic chip. For magnetomotive LSI experiments with a dc magnetic field, a commercially available microfluidic slide (Model SCS0105, thinXXS Microtechnology AG, Zweibrücken, Germany) was used as the test substrate. The channel had a cross section of $300 \mu \mathrm{m}$ throughout its entire length. With a syringe-based displacement pump (Pump 11 Plus, Harvard Apparatus, Holliston, Massachusetts), a mixture of rabbit blood infused with heparin (anticoagulant) and the SPIO nanoparticles (10:1 ratio by volume) was pumped through the channel. Software written in LabVIEW (Version 7.1, National Instruments, Austin, Texas) was used to control the pump.

Iron oxide nanoparticles. Colloidal suspensions of superparamagnetic iron oxide (SPIO) nanoparticles are tissuespecific MRI contrast agents approved in 1996 by the United States Food and Drug Administration (FDA) for human use. Mean core diameter of these particles is $20 \mathrm{~nm}$ and total aggregation diameter is about $100 \mathrm{~nm}$. SPIO nanoparticles consist of nonstoichiometric magnetite crystalline cores, iron, and a dextran T-10 coating that is used to prevent aggregation and provide stabilization in the liver. Feridex I.V. SPIO nanoparticles (Advanced Magnetics, Incorporated, Cambridge, Massachusetts) with a 5-nm core diameter and dextran coating giving a nominal $100 \mathrm{~nm}$ diameter were used in all experiments. The prepared nanoparticle solution consisted of $50-\mathrm{mL}$ $5 \%$ dextrose solution and $1-\mathrm{mL}$ pure Feridex I.V. with a concentration of $0.67 \quad 10^{12}$ iron particles/ $\mu \mathrm{L} \quad(1.12-\mu \mathrm{g}$ $\operatorname{iron} / \mu \mathrm{L}){ }^{28}$

Magnet. A solenoid coil (manufacturer Ledex, part number $4 \mathrm{EF})$ with a cone-shaped ferrite core at the center and driven by a current amplifier supplying up to $960 \mathrm{~W}$ was placed underneath the sample during LSI. The combination of the core and solenoid, using high power operation, dramatically increased the magnetic field strength $\left(B_{\max }=1 \mathrm{~T}\right.$ and field gradient of $220 \mathrm{~T} / \mathrm{m}$ ) at the tip of the core, and also focused the magnetic force on the targeted samples. The magnetic force applied to the capillary tube was varied by a sinusoidal current to induce SPIO nanoparticle movement.

\subsection{Methods}

\subsubsection{General data collection protocol}

After adjusting the pump settings, we activated the pump. After $\sim 10 \mathrm{~s}$, we manually initiated collection of a sequence of raw speckle images. Based on preliminary experiments, we determined empirically that $10 \mathrm{~s}$ was a sufficient period of time to achieve a steady flow rate.

\subsubsection{Magnetomotive laser speckle imaging}

For these experiments, we used a solution of SPIO nanoparticles as the medium, and we fixed the image exposure time at $10 \mathrm{~ms}$. Prior to activation of the magnet, we collected a se- 

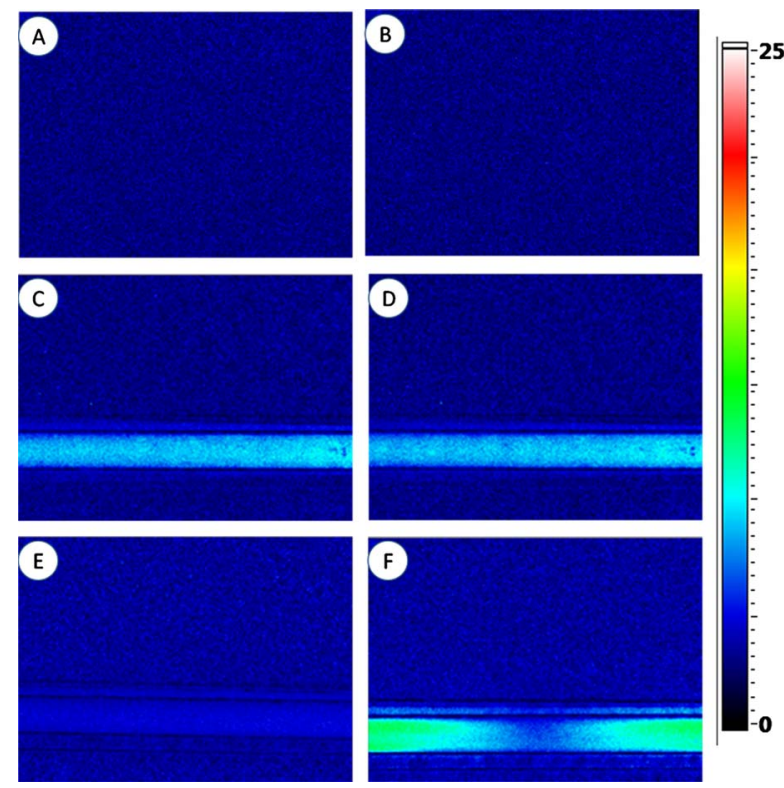

Fig. 2 An ac magnetic field can increase the apparent SFI of an otherwise stagnant solution of SPIO particles. Negative control experiments involving (a) and (b) paper and (c) and (d) milk demonstrate that activation of the magnet alone is insufficient to change the apparent SFI. (e) and (f) Experiments with SPIO particles demonstrate that the apparent SFI increases with application of the oscillating magnetic field.

quence of ten raw speckle images as described before. After manual activation of the magnetic field, we collected a second sequence of ten raw speckle images.

\subsubsection{Data analysis}

After data collection, each image was converted to a speckle contrast image using a $7 \times 7$ sliding window operator, as described previously. ${ }^{29}$ With the simplified speckle imaging equation presented independently by both Cheng and Duong ${ }^{30}$ and Ramirez-San-Juan et al., ${ }^{31}$ each speckle contrast image was converted to a speckle flow index (SFI) map:

$$
\operatorname{SFI}(i, j)=\frac{1}{\tau_{c}}=\frac{1}{T C(i, j)^{2}},
$$

where $(i, j)$ are the pixel coordinates in the image, $\tau_{c}$ is the speckle decorrelation time $[\mathrm{s}], T$ is the exposure time $[\mathrm{s}]$, and $C(i, j)$ is the speckle contrast at pixel $(i, j)$. Briers ${ }^{7}$ proposed that $\tau_{c}$ is equivalent to the correlation time extracted from dynamic light scattering measurements. Dunn et al. ${ }^{1}$ present empirical data that strongly suggest that SFI values are equivalent to the perfusion units associated with laser Doppler flowmetry. The data presented in this work represent an average of 10 SFI images.

\section{Results and Discussion}

Application of an ac magnetic field to a piece of paper [Figs. 2(a) and 2(b)] or to a solution of milk [Figs. 2(c) and 2(d)] does not have a perceivable effect on the SFI images. In contrast, with application of an ac magnetic field to a solution of stagnant SPIO particles, an apparent increase in SFI can be

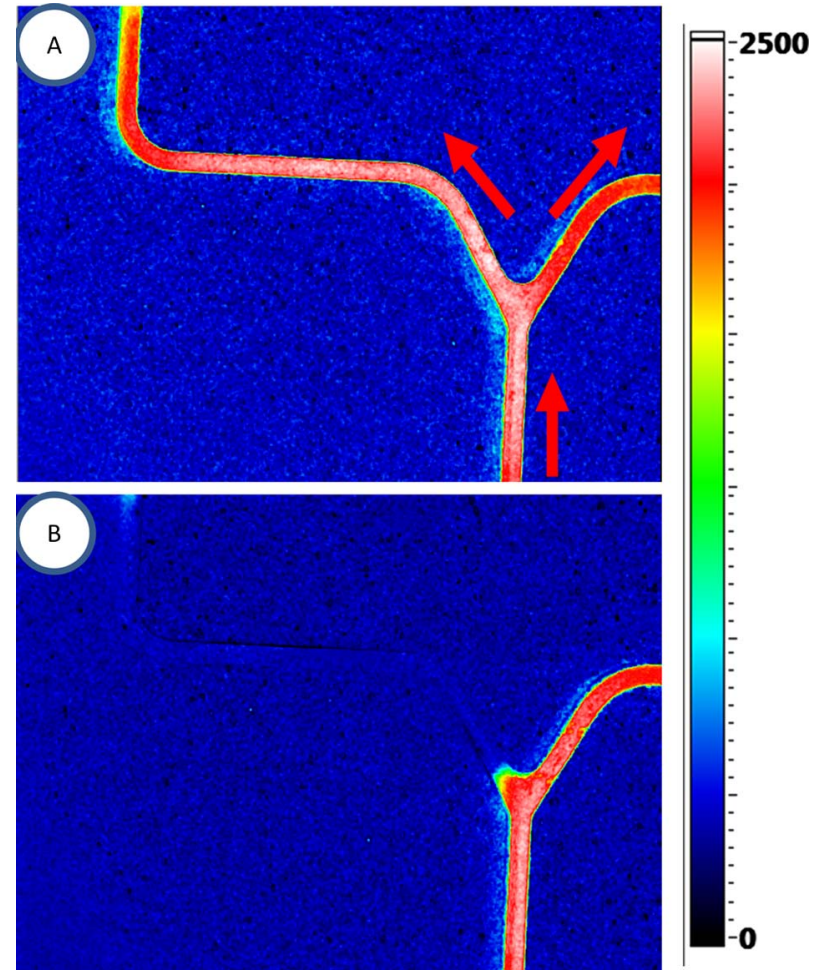

Fig. 3 A dc magnetic field can decrease ordered SPIO particle motion in an in vitro flow phantom. (a) In the absence of the magnetic field SPIO particle flow is observed in both branches of the Y-shaped microfluidic channel. (b) After application of a dc magnetic field for less than one second, a marked decrease is observed in SPIO particle flow through the left portion of the Y-shaped channel.

induced [Figs 2(e) and 2(f)]. With our specific configuration, we observed a $\sim 3 \times$ increase in SFI values after the magnet is activated. A nonuniform SFI pattern was observed because the magnetic field intensity is inversely proportional to the square of the distance between the tip of the probe and a specified lateral position away from the tip.

With application of a focused dc magnetic field, a decrease in SFI can be induced. Prior to activation of the magnet, ordered flow (volumetric flow rate of $1 \mathrm{~mL} / \mathrm{min}$ ) of the SPIO solution was evident in both branches of the Y-shaped channel [Fig. 3(a)]. After activation of a dc magnetic field within the left-hand branch, the SFI values in that branch were reduced to the level of the background [Fig. 3(b)]. The SFI values in the right-hand branch increased by only $\sim 20 \%$, which was unexpectedly low, given the relatively large decrease in SFI values in the left-hand branch. We currently postulate that this unexpected finding may be due to one of several phenomena, including perturbation of the ordered flow velocity distribution in the right-hand branch and/or increased flow resistance in the microfluidic chip, leading to suboptimal performance of the infusion pump and hence lower-than-expected flow rates.

Collectively, our in vitro experimental data demonstrate that magnetic activation of nanoparticles can modulate speckle contrast values, and hence SFI values. Specifically, ac and dc magnetic fields can increase (Fig. 2) and decrease (Fig. $3)$, respectively, SFI values.

These results have broader implications for in vivo diagnostic and therapeutic applications. Oh et al. previously dem- 
onstrated that magnetic activation of monocrystalline iron oxide nanoparticles contained within macrophages can enhance ultrasound $^{21}$ and OCT image contrast. ${ }^{22}$ With judicious selection of magnetic field parameters, it may be possible to enhance wide-field speckle contrast and hence detectability of tumors. Tumor blood flow typically is sluggish and thus is capable of inducing only a minor decrease at best in speckle contrast. With infusion of SPIO nanoparticles into the circulatory system and subsequent activation with an ac magnetic field, we expect an appreciable decrease in speckle contrast would result. Use of surface markers, such as monoclonal antibodies, to enable molecular targeting of specific receptors (i.e., epidermal growth factor receptor ${ }^{25}$ ) is expected to provide a mechanism for SPIO or similar nanoparticles to localize specifically within cancer cells, and hence provide an additional source of specific speckle contrast reduction with magnetic activation. Furthermore, with both lateral and longitudinal translation of the applied ac magnetic field, it may be possible to achieve some degree of 3-D mapping of tissue blood flow.

With application of a dc magnetic field, we were able to shut down flow in one branch of a microfluidic channel (Fig. 3 ). It may be possible to use this approach to improve on light-based strategies designed to induce selective coagulation of blood vessels. For example, after infusion of the circulation with SPIO nanoparticles, a clinician could use a combination therapy protocol employing light-based therapy [i.e., pulsed dye laser (PDL) or photodynamic therapy] with preceding or ensuing activation of the SPIO particles with a dc magnetic field. Vargas, Barton, and Welch ${ }^{32}$ have demonstrated that a combined glycerol-PDL therapeutic protocol results in significantly enhanced acute shutdown of blood flow. Several previous studies ${ }^{33,34}$ including one from our group, ${ }^{5}$ have demonstrated that glycerol diminishes considerably blood flow in arterioles, venules, and capillaries. Hence, we expect to observe a similar outcome with SPIO particle activation.

We previously have demonstrated that magnetic activation of hemoglobin ${ }^{23}$ and SPIO nanoparticles ${ }^{24}$ can enhance the vascular contrast of Doppler optical coherence tomography. Although activation of hemoglobin is attractive due to its endogenous nature, activation of exogenous SPIO nanoparticles is expected to be a more feasible method for in vivo applications due to the large difference in magnetic susceptibility between hemoglobin and SPIO $\left(10^{-5}\right.$ and 1 , respectively). ${ }^{19}$ Since LSI is a wide-field imaging modality, the combination of it with activation of intravascular SPIO nanoparticles may enable lateral mapping of suspicious tissue regions, followed by high-resolution optical interrogation of these regions, as a form of image-guided microscopy. Subsequent photoactivation of the intravascular SPIO nanoparticles could then be performed to induce selective photothermal destruction of unwanted vasculature. ${ }^{35}$ Further in vitro optimization studies and in vivo studies of magnetomotive LSI are warranted.

\section{Acknowledgments}

This work was supported in part by the Arnold and Mabel Beckman Foundation, the A. Ward Ford Foundation, the National Institutes of Health Laser Microbeam and Medical Program (LAMMP, a P41 Technology Research Resource), the Fundamental Science Research Fund (R01-2007-000-2056-0), and the Research and Development on Oriental Medicine Treatment Technology funded by the Ministry of Health, Welfare, and Family Affairs (B080033).

\section{References}

1. A. K. Dunn, T. Bolay, M. A. Moskowitz, and D. A. Boas, "Dynamic imaging of cerebral blood flow using laser speckle," J. Cereb. Blood Flow Metab. 21, 195-201 (2001).

2. H. Y. Cheng, Q. M. Luo, S. Q. Zeng, S. B. Chen, J. Cen, and H. Gong, "Modified laser speckle imaging method with improved spatial resolution," J. Biomed. Opt. 8(3), 559-564 (2003).

3. T. M. Le, J. S. Paul, H. Al-Nashash, A. Tan, A. R. Luft, F. S. Sheu, and S. H. Ong, "New insights into image processing of cortical blood flow monitors using laser speckle imaging," IEEE Trans. Med. Imaging 26, 833-842 (2007).

4. D. D. Duncan and S. J. Kirkpatrick, "Can laser speckle flowmetry be made a quantitative tool?" Opt. Lett. 25, 2088-2094 (2008).

5. B. Choi, J. C. Ramirez-San-Juan, J. Lotfi, and J. S. Nelson, "Linear response range characterization and in vivo application of laser speckle imaging of blood flow dynamics," J. Biomed. Opt. 11, 041129 (2006).

6. A. B. Parthasarathy, W. J. Tom, A. Gopal, X. J. Zhang, and A. K. Dunn, "Robust flow measurement with multi-exposure speckle imaging," Opt. Express 16, 1975-1989 (2008).

7. J. D. Briers, "Laser Doppler and time-varying speckle: a reconciliation," J. Opt. Soc. Am. A 13, 345-350 (1996).

8. Y. C. Huang, T. L. Ringold, J. S. Nelson, and B. Choi, "Noninvasive blood flow imaging for real-time feedback during laser therapy of port wine stain birthmarks," Lasers Surg. Med. 40, 167-173 (2008).

9. W. C. Jia, B. Choi, W. Franco, J. Lotfi, B. Majaron, G. Aguilar, and J. S. Nelson, "Treatment of cutaneous vascular lesions using multiple-intermittent cryogen spurts and two-wavelength laser pulses: numerical and animal studies," Lasers Surg. Med. 39, 494-503 (2007).

10. B. Choi, W. C. Jia, J. Channual, K. M. Kelly, and J. Lotfi, "The importance of long-term monitoring to evaluate the microvascular response to light-based therapies," J. Invest. Dermatol. 128, 485-488 (2008).

11. W. H. Luo, Z. Wang, P. C. Li, S. Q. Zeng, and Q. M. Luo, "A modified mini-stroke model with region-directed reperfusion in rat cortex," J. Cereb. Blood Flow Metab. 28, 973-983 (2008).

12. H. K. Shin, M. Nishimura, P. B. Jones, H. Ay, D. A. Boas, M. A. Moskowitz, and C. Ayata, "Mild induced hypertension improves blood flow and oxygen metabolism in transient focal cerebral ischemia," Stroke 39, 1548-1555 (2008).

13. D. Zhu, J. Zhang, H. Cui, Z. Z. Mao, P. C. Li, and Q. M. Luo, "Short-term and long-term effects of optical clearing agents on blood vessels in chick chorioallantoic membrane," J. Biomed. Opt. 13 , 021106 (2008).

14. S. J. Kirkpatrick, D. D. Duncan, R. K. Wang, and M. T. Hinds, "Quantitative temporal speckle contrast imaging for tissue mechanics," J. Opt. Soc. Am. A 24, 3728-3734 (2007).

15. Z. P. Chen, T. E. Milner, D. Dave, and J. S. Nelson, "Optical Doppler tomographic imaging of fluid flow velocity in highly scattering media," Opt. Lett. 22, 64-66 (1997).

16. J. K. Barton, A. J. Welch, and J. A. Izatt, "Investigating pulsed dye laser-blood vessel interaction with color Doppler optical coherence tomography," Opt. Express 3, 251-256 (1998).

17. D. A. Boas and A. G. Yodh, "Spatially varying dynamical properties of turbid media probed with diffusing temporal light correlation," $J$. Opt. Soc. Am. A Opt. Image Sci. Vis 14, 192-215 (1997).

18. J. N. Anker and R. Kopelman, "Magnetically modulated optical nanoprobes," Appl. Phys. Lett. 82, 1102-1104 (2003).

19. A. L. Oldenburg, J. R. Gunther, and S. A. Boppart, "Imaging magnetically labeled cells with magnetomotive optical coherence tomography," Opt. Lett. 30, 747-749 (2005).

20. A. L. Oldenburg, F. J. J. Toublan, K. S. Suslick, A. Wei, and S. A. Boppart, "Magnetomotive contrast for in vivo optical coherence tomography," Opt. Express 13, 6597-6614 (2005).

21. J. Oh, M. D. Feldman, J. Kim, C. Condit, S. Emelianov, and T. E. Milner, "Detection of magnetic nanoparticles in tissue using magneto-motive ultrasound," Nanotechnology 17, 4183-4190 (2006).

22. J. Oh, M. D. Feldman, J. Kim, H. W. Kang, P. Sanghi, and T. E. Milner, "Magneto-motive detection of tissue-based macrophages by 
differential phase optical coherence tomography," Lasers Surg. Med. 39, 266-272 (2007).

23. J. Kim, J. Oh, T. E. Milner, and J. S. Nelson, "Hemoglobin contrast in magnetomotive optical Doppler tomography," Opt. Lett. 31, 778-780 (2006).

24. J. Kim, J. Oh, T. E. Milner, and J. S. Nelson, "Imaging nanoparticle flow using magneto-motive optical Doppler tomography," Nanotechnology 18, 035504 (2007).

25. J. S. Aaron, J. Oh, T. A. Larson, S. Kumar, T. E. Milner, and K. V. Sokolov, "Increased optical contrast in imaging of epidermal growth factor receptor using magnetically actuated hybrid gold/iron oxide nanoparticles," Opt. Express 14, 12930-12943 (2006).

26. Y. Jing, N. Mal, P. S. Williams, M. Mayorga, M. S. Penn, J. J. Chalmers, and M. Zborowski, "Quantitative intracellular magnetic nanoparticle uptake measured by live cell magnetophoresis," FASEB J. 22, 4239-4247 (2008).

27. S. J. Kirkpatrick, D. D. Duncan, and E. M. Wells-Gray, "Detrimental effects of speckle-pixel size matching in laser speckle contrast imaging," Opt. Lett. 33, 2886-2888 (2008).

28. D. Artemov, "Molecular magnetic resonance imaging with targeted contrast agents," J. Cell. Biochem. 90, 518-524 (2003).

29. J. D. Briers, G. Richards, and X. W. He, "Capillary blood flow monitoring using laser speckle contrast analysis (LASCA)," J. Biomed. Opt. 4, 164-175 (1999).
30. H. Y. Cheng and T. Q. Duong, "Simplified laser-speckle-imaging analysis method and its application to retinal blood flow imaging," Opt. Lett. 32, 2188-2190 (2007).

31. J. C. Ramirez-San-Juan, R. Ramos-Garcia, I. Guizar-Iturbide, G. Martinez-Niconoff, and B. Choi, "Impact of velocity distribution assumption on simplified laser speckle imaging equation," Opt. Express 16, 3197-3203 (2008).

32. G. Vargas, J. K. Barton, and A. J. Welch, "Use of hyperosmotic chemical agent to improve the laser treatment of cutaneous vascular lesions," J. Biomed. Opt. 13, 021114 (2008).

33. E. I. Galanzha, V. V. Tuchin, A. V. Solovieva, T. V. Stepanova, Q. Luo, and H. Cheng, "Skin backreflectance and microvascular system functioning at the action of osmotic agents," J. Phys. D: Appl. Phys. 36, 1739-1746 (2003).

34. G. Vargas, A. Readinger, S. S. Dozier, and A. J. Welch, "Morphological changes in blood vessels produced by hyperosmotic agents and measured by optical coherence tomography," Photochem. Photobiol. 77, 541-549 (2003).

35. J. Kim, J. Oh, H. W. Kang, M. D. Feldman, and T. E. Milner, "Photothermal response of superparamagnetic iron oxide nanoparticles," Lasers Surg. Med. 40, 415-421 (2008). 